\title{
Bupivacaine Ipsilateral Interscalene Block-Induced Localized Hypoxic Brain Injuries via Vasoconstriction
}

\author{
Min-Jun Song, MD (D); Hee-Jin Im, MD, PhD (D) \\ Department of Neurology, Dongtan Sacred Heart Hospital, Hallym University College of Medicine, Hwaseong, Korea
}

Local anesthetic systemic toxicity is a rare but serious consequence that occurs when a local anesthetic is introduced into the plasma leading to neurological or cardiac toxic complications. Rich vasculature such as the internal carotid artery, which is adjacent to the interscalene block site, allows hazards to direct unintentional intravascular injections or indirect fast absorption to the regional vascular system. We present an interesting case of a patient who-after interscalene brachial plexus block using bupivacaine-presented with localized ipsilateral cortical hypoxic injury by vasoconstriction of a distal arteriole of the middle cerebral artery which resulted in postoperative focal seizure and severe hemiplegia.

J Neurosonol Neuroimag 2020;12(2):\$7-go

Key Words: Local anesthetic systemic toxicity; Bupivacaine; Vascoconstriction; Hypoxic brain damage; Interscalene anesthesia
Received: April 28, 2020

Revised: July 7, 2020

Accepted: August 1, 2020

Correspondence:

Hee-Jin Im, MD, PhD

Department of Neurology, Dongtan Sacred Heart Hospital, Hallym University College of Medicine, 7 Keunjaebong-gil, Hwaseong 18450 , Korea Tel: $+82-31-8680-3185$ Fax: $+82-31-8680-2317$ E-mail: coolere@naver.com
Local anesthetic systemic toxicity is a rare but serious critical event occurring when local anesthesia is introduced into the plasma leading to neurological or cardiac toxic complications. ${ }^{1}$ Brachial plexus block with local anesthetics via the interscalene approach is a widely performed procedure for shoulder surgery. Rich vasculature such as the internal carotid artery, which is adjacent to the interscalene block site, allow hazards to direct unintentional intravascular injections or indirect fast absorption to the regional vascular system. Transient central nervous system (CNS) complications such as generalized seizures after local ropivacaine or bupivacaine administration have been demonstrated earlier, ${ }^{2-4}$ but there has been no evidence of permanent and serious disability or localized injury. We introduce an interesting case of a patient who after interscalene brachial plexus block using bupivacaine presented with localized ipsilateral cortical hypoxic injury by vasoconstriction which resulted in focal seizures and severe hemiplegia postoperatively.

\section{CASE}

A 54-year-old previously healthy male was transferred for complete left hemiplegia and gaze preference towards the right at 4 hours from symptom onset, which developed after arthroscopic rotator cuff repair surgery on his right shoulder. General anesthesia was induced with propofol (120 mg) and lidocaine (40 $\mathrm{mg}$ ), and was maintained with inhaled sevoflurane and nitrous oxide in oxygen via a laryngeal mask airway. A total volume of $13 \mathrm{mg}$ of $0.5 \%$ bupivacaine mixed with $12 \mathrm{~mL}$ of saline was used for interscalene brachial plexus block on the right side. There was no intraoperative hemodynamic instability based on the surgical report. The initial vital signs of the patient were stable and electrocardiography revealed sinus rhythm at the emergency room. Conventional computed tomography (CT) angiography showed no definite stenosis or occlusion of the right middle cerebral artery (MCA) (Fig. 1A) and perfusion CT revealed relatively delayed mean transit time and decreased cerebral blood flow and preserved blood volume in the anterior cortical border zone between the supply 

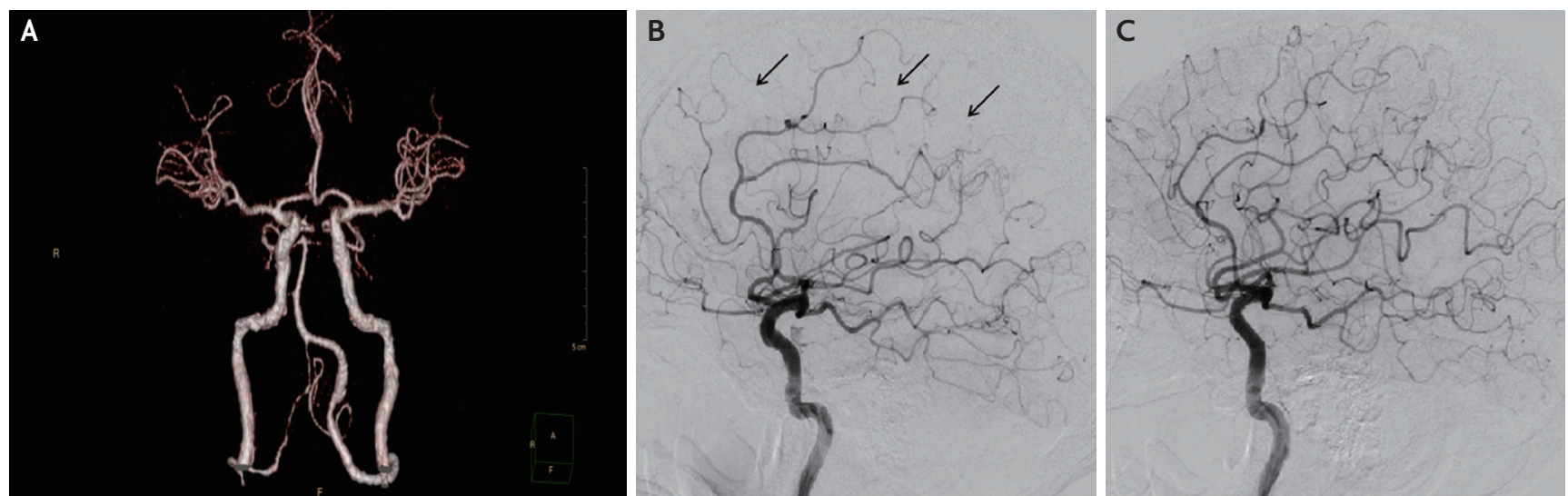

FIG. 1. (A) Cerebral angiography by conventional computed tomography showed no definite stenosis of the right middle cerebral artery (MCA) but (B) transfemoral cerebral angiography revealed decreased filling and narrow diameter of the distal cortical arteriole and decreased perfusion (arrows) of the right MCA territory (C) compared with the other side, the left MCA.
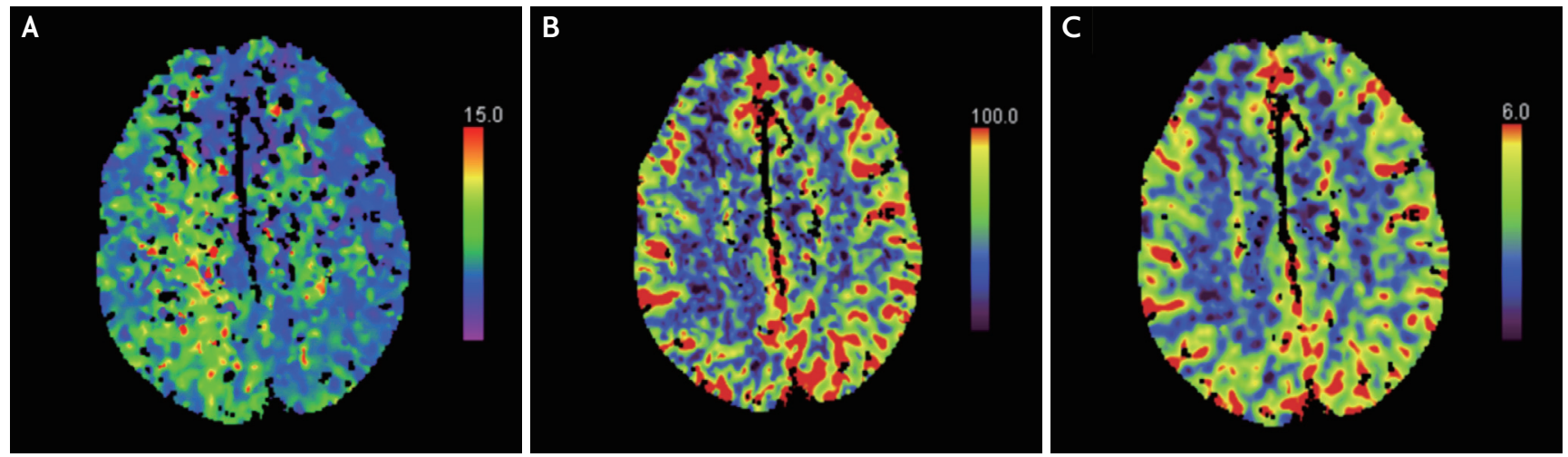

FIG. 2. Perfusion computed tomography revealed decreased blood supply restricted in the cortical border zone between the right anterior cerebral artery and middle cerebral artery (external border zone area). (A) Time to peak mapping. (B) Blood flow mapping. (C) Blood volume mapping.
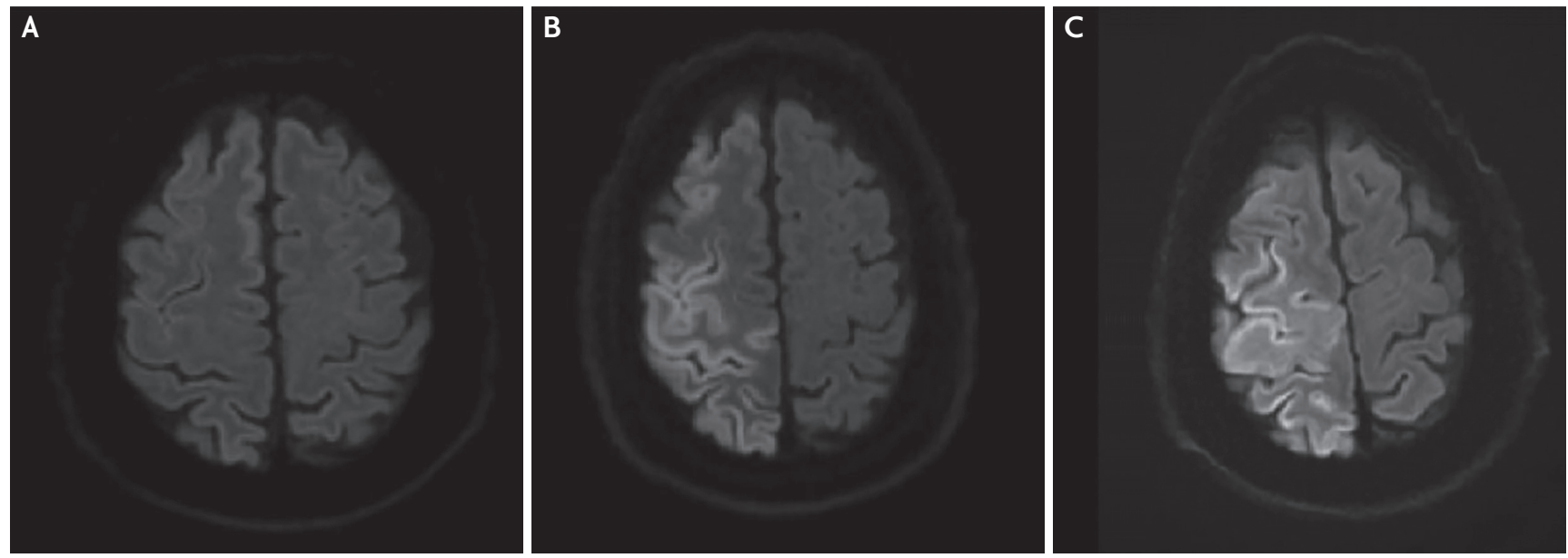

FIG. 3. Serial diffusion-weighted magnetic resonance imaging showed delayed evolution of localized cortical injury with perilesional cytotoxic edema which is supplied by the right intracerebral artery. (A) Four hours after symptom onset. (B) Three days after symptom onset. (C) Ten days after symptom onset. 
territory of the right MCA and anterior cerebral artery (Fig. 2). There was no diffusion restricted lesion in diffusion weighted (DW) magnetic resonance imaging (MRI) (Fig. 3A). Considering the benefit from reperfusion therapy due to diffusion-perfusion mismatch, a transfemoral cerebral angiography was performed which revealed mildly narrowing, delayed filling of the distal cortical arteriole and relatively scanty perfusion on the right MCA territory compared to the contralateral side without steno-occlusion (Fig. 1B, C). Serial follow-up DW MRI demonstrated more prominent localized cortical cytotoxic injuries in the right intracerebral artery territory (Fig. 3). Nine hours after symptom onset, the patient began to experience focal motor seizures, presenting as facial twitching on his left side. Electroencephalography confirmed that the epileptic focus originated in the right parieto-occipital area. After the administration of antiepileptic drugs (valproic acid 1,800 mg, levetiracetam 2,000 mg, and phenytoin 350 $\mathrm{mg} /$ day) and nimodipine, the mental status and paralysis on the left leg of the patient were rapidly improved. The exploration for an embolic source such as transesophageal cardiac echography and Holter monitoring revealed no abnormal findings. Qualitative screening of bupivacaine by mass spectrograph was conducted 22 hours postoperatively and free-form bupivacaine was identified in the serum. Upon discharge, he could walk with assisted gait but could hardly move his left hand (Medical Research Council grade 1) resulting in neurological sequelae (modified Rankin Scale 3). Informed consent was obtained from the patient in this case for identifiable images or other information within the manuscript.

\section{DISCUSSION}

This is an interesting case presenting permanent localized hypoxic injury of the CNS followed by partial status epilepticus after ipsilateral injection of bupivacaine through the interscalene approach. Although there have been more reports of generalized seizures after local injection of bupivacaine by interscalene than by other approaches, ${ }^{1}$ reports on localized injury of the CNS or focal seizures due to toxicity of local anesthetics are rare, and no reports on serious sequelae after such events are available. The close proximity of a high vascular supply during the interscalene approach can lead to unintended vascular injury or fast absorption and result in consequent toxic reactions either directly or indirectly. ${ }^{5}$ The influence on vascular tone as potent vasoconstrictors of amide local anesthetics like lidocaine and bupivacaine has been reported. This was proven by reduced blood flow of the epidural arteries after epidural block, ${ }^{6}$ and impaired pial microcirculation after injection of ropivacaine by pial vessel constriction in canine cases. ${ }^{7}$ The dose-dependent arteriolar vasoconstriction of bupivacaine on the microvasculature when both intravenously and topically applied was reported in an in vivo rat study. ${ }^{8}$ The electroencephalography finding of our case was a generation of focal ictal discharge from the damaged cortical neurons in the hypoxic area. A similar case had been reported, demonstrating focal motor seizures on the arm of the contralateral side after interscalene brachial plexus block using ropivacaine. ${ }^{7}$ Considering the possibility of unintentional direct intra-arterial injection of bupivacaine into the ipsilateral carotid artery due to the adjacent anatomical approach of the interscalene procedure, the critical limitation of this hypothesis is that free plasma concentration of bupivacaine was not evaluated due to lack of an appropriate facility. Qualitative detection of bupivacaine by mass spectrograph was performed relatively late (after about nine times of half-life), but the freeform of bupivacaine was still present in the serum. Considering its high affinity to bind protein (95\%) and short half-life time (2.7 hours), it may support longterm toxic effects on the CNS. ${ }^{9}$ In addition, it is a limitation that due to loss of follow-up, there was no further clinical or radiological information on the patient after discharge for evaluating the effectiveness of nimodipine, reversibility of vasoconstriction, or the long term neurological outcome. The present case, however, elucidates concerns of local amide anesthetics such as bupivacaine, as a potent vasoconstrictor, supported by imaging modalities and serological confirmation.

\section{Conflicts of interest}

No potential conflicts of interest relevant to this article was reported. 


\section{REFERENCES}

1. Brown DL, Ransom DM, Hall JA, Leicht CH, Schroeder DR, Offord KP. Regional anesthesia and local anesthetic-induced systemic toxicity: seizure frequency and accompanying cardiovascular changes. Anesth Analg. 1995;81:321-328.

2. Agarwal R, Gutlove DP, Lockhart CH. Seizures occurring in pediatric patients receiving continuous infusion of bupivacaine. Anesth Analg. 1992;75:284-286.

3. Abouleish EI, Elias M, Nelson C. Ropivacaine-induced seizure after extradural anaesthesia. Br J Anaesth. 1998;80:843844.

4. Güngör İ, Akbaş B, Kaya K, Çelebi H, Tamer U. Sudden developing convulsion during interscalene block: does propofol anesthesia diminish plasma bupivacaine level?. Agri. 2015;27:54-57.
5. Nishiyama T, Komatsu K. Local anesthetic toxicity in interscalene block: clinical series. Minerva Anestesiol. 2010;76:1088-1090.

6. Dahl JB, Simonsen L, Mogensen T, Henriksen JH, Kehlet H. The effect of $0.5 \%$ ropivacaine on epidural blood flow. Acta Anaesthesiol Scand. 1990;34:308-310.

7. Ishiyama T, Dohi S, Iida H, Watanabe Y. The effects of topical and intravenous ropivacaine on canine pial microcirculation. Anesth Analg. 1997;85:75-81.

8. Johns RA, Seyde WC, DiFazio CA, Longnecker DE. Dose-dependent effects of bupivacaine on rat muscle arterioles. Anesthesiology. 1986;65:186-191.

9. Wilkinson GR, Lund PC. Bupivacaine levels in plasma and cerebrospinal fluid following peridural administration. Anesthesiology. 1970;33:482-486. 